\title{
Reactions of Fluorinated Nitriles with Sulphur(II) Chlorides; Crystal Structure of 2,4,6-Tris(difluorosulphoximido)-1,3,5-triazene at $170 \mathrm{~K}$
}

\author{
Hans-Ulrich Höfs, Rüdiger Mews*, Mathias Noltemeyer, George M. Sheldrick, and Martin Schmidt \\ Institut für Anorganische Chemie der Universität, Tammannstraße 4, D-3400 Göttingen \\ Gerald Henkel and Bernt Krebs \\ Institut für Anorganische Chemie der Universität, Gievenbecker Weg 9, D-4400 Münster
}

Z. Naturforsch. 38b, 454-459 (1983); eingegangen am 8. November 1982

Crystal Structure, Perfluoromethanesulphenylformylimidoylchlorides, N-Sulphenylchlorides, 1,3,5-Triazenes

The addition of $\mathrm{CF}_{3} \mathrm{SCl}(\mathbf{2})$ to the fluorinated nitriles $\mathrm{R}_{\mathrm{f}} \mathrm{CN}\left[\mathrm{R}_{\mathrm{f}}=\mathrm{CF}_{3}(\mathbf{3}), \mathrm{NSF}_{2}(4), \mathrm{NSOF}_{2}\right.$ (5)] leads to N-perfluoromethanesulphenyl-formylimidoylchlorides $\mathrm{CF}_{3} \mathrm{~S}-\mathrm{N}=\mathrm{C}(\mathrm{Cl}) R_{f}$ $(6,11,12)$. From the reactions of $\mathrm{SCl}_{2}(\mathbf{1})$ with $(\mathbf{3})$ and $(\mathbf{5})$, which yielded the $\mathrm{N}$-sulphenylchlorides $\mathrm{ClS}-\mathrm{N}=\mathrm{C}(\mathrm{Cl}) R_{f}(\mathbf{7}, \mathbf{1 3})$, it proved possible also to isolate the oxidation product $\left[\mathrm{CF}_{3} \mathrm{CCl}_{2} \mathrm{NSCl}_{2}(8)\right]$ and an oligomer of the nitrile $\left[\left(\mathrm{NCNSOF}_{2}\right)_{3}(\mathbf{1 4})\right]$. Dechlorination of 7 with $\mathrm{Hg}$ gives the corresponding di- and tri-sulphides $\left(\mathrm{CF}_{3} \mathrm{C}(\mathrm{Cl})=\mathrm{N}-\right)_{2} \mathrm{~S}_{x}(\mathbf{9}, \mathbf{1 0}, x=2,3)$. Crystals of (14) are triclinic with $a=1133.6(5), b=1332.6(5), c=1715.1(8) \mathrm{pm}, \alpha=$ $109.31(3), \beta=105.30(3), \gamma=97.84(3)^{\circ}$ at $170 \mathrm{~K}, Z=8$, space group $\mathrm{P} \overline{\mathrm{l}}$. The structure was refined to $R=0.028$ for 8290 diffractometer data with $\mathrm{F}>4 \sigma(\mathrm{F})$. The four crystallographically independent molecules adopt similar unsymmetrical conformations.

N-Sulphenylacetimidoyl halides of the type $\mathrm{RC}(\mathrm{X})=\mathrm{N}-\mathrm{S}-\mathrm{Y}$ are important in preparative chemistry for several reasons: (a) When $\mathrm{Y}$ is a halogen, it may be readily exchanged, leading to a chemistry analogous to that of the perhaloalkylsulfenylhalides $\mathrm{R}_{\text {hal }} \mathrm{SCl}[1,2]$. Examples of such substitution reactions have already been reported $[2,3]$. (b) When the substituent $\mathrm{X}$ on the triply-coordinated carbon is a halogen, the $-\mathrm{M}$ effect of the imino-nitrogen facilitates nucleophilic attack. Hard nucleophiles (fluoride, alcohols, amines) tend to replace X [4], soft nucleophiles the sulphur-substituent $Y$. (c) The $>\mathrm{C}=\mathrm{N}$-S-system may be regarded as a shortened diene, in which the second double bond is replaced by the oxidisable sulphur atom; instead of the usual 1,4 -addition this results in 1,3-addition with simultaneous oxidation of the sulphur [5].

One of the simplest examples of this class of compounds, chlorosulphenylchloroformylimidoylchloride may be prepared in several ways; the synthesis [6] from $\mathrm{ClCN}$ and $\mathrm{SCl}_{2}$ opens a general route to the $\mathrm{N}$-sulphenylimidoyl derivatives [4, 7-9]:

$$
\begin{gathered}
\mathrm{ClCN}+\mathrm{SCl}_{2} \rightarrow \mathrm{ClS}-\mathrm{N}=\mathrm{CCl}_{2} \\
\mathbf{1} \\
\mathrm{RCN}+\mathrm{R}^{\prime} \mathrm{SCl} \rightarrow \mathrm{R}^{\prime} \mathrm{S}-\mathrm{N}=\mathrm{CClR} \\
\mathbf{2}
\end{gathered}
$$

\footnotetext{
* Reprint requests to Prof. R. Mews. 0340-5087/83/0400-0454/\$01.00/0
}

We report here an investigation of the corresponding addition of $\mathrm{CF}_{3} \mathrm{SCl}$ and $\mathrm{SCl}_{2}$ to the fluorinated nitriles $\mathrm{N} \equiv \mathrm{C}-\mathrm{CF}_{3}, \mathrm{~N} \equiv \mathrm{C}-\mathrm{NSF}_{2}$ and $\mathrm{N} \equiv \mathrm{C}-\mathrm{NSOF}_{2}$.

\section{Experimental}

Starting materials were prepared according to literature methods: $\mathrm{SCl}_{2}$ (1) [10], $\mathrm{CF}_{3} \mathrm{SCl}(2)$ [11], $\mathrm{CF}_{3} \mathrm{CN}$ (3) [12], $\mathrm{NCNSF}_{2}$ (4) [13], and $\mathrm{NCNSOF}_{2}(5)$ [14]. IR spectra were recorded on Perkin-Elmer 325 and 180 grating spectrometers, mass-spectra with a Varian MAT CH5 instrument at $70 \mathrm{eV}$ ionisation voltage. $\mathrm{CFCl}_{3}$ was employed as solvent and internal standard for the ${ }^{19} \mathrm{~F}$ NMR measurements, which were obtained on a Bruker $60 \mathrm{E}$ spectrometer. Vapour pressure and derived data were calculated from about 10 data points per sample.

\section{Preparation of $\mathrm{CF}_{3} \mathrm{C}(\mathrm{Cl})=\mathrm{N}-\mathrm{SCF}_{3}(6)$}

$15.8 \mathrm{~g}(0.166 \mathrm{~mol}) 3$ and $21.9 \mathrm{~g}(0.160 \mathrm{~mol}) 2$ were condensed on $c a .0 .5 \mathrm{~g}$ active carbon in a steel autoclave. After shaking for $5 \mathrm{~d}$ at $20^{\circ} \mathrm{C}$, the volatile products were fractionated under oil-pump vacuum. 6 condensed in the $-95^{\circ} \mathrm{C}$ trap; the IR and ${ }^{19} \mathrm{~F}$ NMR spectra were identical to those previously reported. Yield $30.5 \mathrm{~g}(0.132 \mathrm{~mol})=82 \%$.

\section{Preparation of $\mathrm{CF}_{3} \mathrm{C}(\mathrm{Cl})=\mathrm{N}-\mathrm{SCl}(\mathbf{7})$}

$3 \mathrm{~g}$ active carbon, $15.8 \mathrm{~g}(0.166 \mathrm{~mol}) 3$ and $20.9 \mathrm{~g}$ $(0.203 \mathrm{~mol}) 1$ were shaken at $40{ }^{\circ} \mathrm{C}$ for $2 \mathrm{~d}$ in a steel autoclave until the pressure dropped to below 3 bar. If the reaction ceases prematurely, the active carbon must be renewed. The mixture was fractionated through a 30-cm-Vigreux-column under rough vacuum. Yield $19.9 \mathrm{~g}(0.101 \mathrm{~mol})=61 \%$. For 
characterisation a sample was freed from traces of 1 by GC $\left(2 \mathrm{~m} \mathrm{SE} 30,80^{\circ} \mathrm{C}\right) .7$ is pale yellow, $\ln \left(\mathrm{P} / \mathrm{P}_{0}\right)=$ $-4956.6 / \mathrm{T}+13.147$, b.p. $103.9^{\circ} \mathrm{C}$ (extr.), $\Delta \mathrm{H}_{\mathrm{v}}=$ $41.21 \mathrm{~kJ} \mathrm{~mol}^{-1}, \Delta \mathrm{S}_{\mathrm{v}}=109.3 \mathrm{~J} \mathrm{~K}^{-1} \mathrm{~mol}^{-1}$.

$\mathrm{C}_{2} \mathrm{Cl}_{2} \mathrm{~F}_{3} \mathrm{NS}(\mathrm{M}=197.99)$

Caled C 12.1 Cl 35.8 F 28.8 N 7.07 S 16.2, Found C $11.6 \mathrm{Cl} 35.3 \mathrm{~F} 27.2 \mathrm{~N} 6.84 \mathrm{~S} 16.1$.

MS $m / e$ (relative intensities in parentheses): $197 \mathrm{M}^{+}(85), 162 \mathrm{M}^{-} \mathrm{Cl}^{+}(100), 128 \mathrm{M}^{-} \mathrm{CF}_{3}{ }^{+}(38)$, 127 (19), $93(24), 69 \mathrm{CF}_{3}+(99), 67 \mathrm{SCl}^{+}(71), 64(35)$, $50(26)$. ${ }^{19} \mathrm{~F}$ NMR: $\delta\left(\mathrm{CF}_{3}\right)=-71.4 \mathrm{ppm}(50 \%$ in $\left.\mathrm{CFCl}_{3}\right)$.

IR (KBr, gas) cm-1: 1671 (w), 1624 (s), 1323 (s), 1290 (vs), 1225 (vs), 1181 (vs), 957 (vs), 864 (s), $798(\mathrm{~m}), 716(\mathrm{~s}), 553(\mathrm{~s}), 476(\mathrm{~s})$.

\section{Preparation of $\mathrm{CF}_{3} \mathrm{CCl}_{2} \mathrm{~N}=\mathrm{SCl}_{2}$ (8)}

$1 \mathrm{~g}$ active carbon, $3.18 \mathrm{~g}$ (33.5 mmol) 3 and $4.04 \mathrm{~g}$ $(39.2 \mathrm{mmol}) 1$ were shaken for $2 \mathrm{~d}$ at $40^{\circ} \mathrm{C}$ in a steel autoclave. A further $9.30 \mathrm{~g}(90.3 \mathrm{mmol})$ of 1 were condensed in, and the mixture shaken for a further $2 \mathrm{~d}$ at $40{ }^{\circ} \mathrm{C}$. The product was fractionated through a 30 -cm -Vigreux-column. Yield $5.10 \mathrm{~g}(19.0 \mathrm{mmol})=$ $57 \% .8$ is pale yellow, $\ln \left(\mathrm{P} / \mathrm{P}_{0}\right)=-5813.9 / \mathrm{T}+$ 13.164, b. p. $=168.5^{\circ} \mathrm{C}$ (extr.), $\Delta \mathrm{H}_{\mathrm{v}}=48.34 \mathrm{~kJ} \mathrm{~mol}^{-1}$, $\Delta \mathrm{S}_{\mathrm{v}}=109.5 \mathrm{~J} \mathrm{~K}^{-1} \mathrm{~mol}^{-1}$.

$\mathrm{C}_{2} \mathrm{Cl}_{4} \mathrm{~F}_{3} \mathrm{NS}(\mathrm{M}=268.96)$

$$
\text { Calcd } \quad \text { C } 8.93 \quad \text { Cl 52.7 } \quad \text { N 5.21 } \quad \text { S 11.9, }
$$$$
\text { Found C 8.92 Cl 52.2 N 5.34 S 12.0. }
$$

${ }^{19} \mathrm{~F} \mathrm{NMR} \delta\left(\mathrm{CF}_{3}\right)=-82.8 \mathrm{ppm}(\mathrm{s})\left(70 \%\right.$ in $\left.\mathrm{CFCl}_{3}\right)$. IR (KBr, gas) $\mathrm{cm}^{-1}: 1315(\mathrm{~s}), 1260(\mathrm{vs}), 1231(\mathrm{~s})$, $1225(\mathrm{~s}), 947(\mathrm{~m}), 916(\mathrm{~m}), 845(\mathrm{~s}), 725(\mathrm{~m}), 570(\mathrm{w})$, $539(\mathrm{w}), 430(\mathrm{~m}), 400(\mathrm{~m})$.

Preparation of $\left[\mathrm{CF}_{3} \mathrm{C}(\mathrm{Cl})=\mathrm{N}-\mathrm{S}-\right]_{2}(\mathbf{9})$ and $\left[\mathrm{CF}_{3} \mathrm{C}(\mathrm{Cl})=\mathrm{N}-\mathrm{S}-\right]_{2} \mathrm{~S}(\mathbf{1 0})$

$70 \mathrm{~g}(0.349 \mathrm{~mol}) \mathrm{Hg}, 14.00 \mathrm{~g}(70.7 \mathrm{mmol}) 7$, and $50 \mathrm{ml} \mathrm{CH}_{2} \mathrm{Cl}_{2}$ were stirred intensively for $10 \mathrm{~d}$ at $20^{\circ} \mathrm{C}$. After filtering and washing through with $\mathrm{CH}_{2} \mathrm{Cl}_{2}$, the solvent was pumped off the filtrate, and the resulting products fractionated through a $30-\mathrm{cm}$ Vigreux-column.

Yields: $96.80 \mathrm{~g}(20.9 \mathrm{mmol})=30 \%, 101.12 \mathrm{~g}$ $(3.1 \mathrm{mmol})=13 \% .9$ is pale yellow, $\ln \left(\mathrm{P} / \mathrm{P}_{0}\right)=$ $-6614.5 / \mathrm{T}+14.649$, b.p. $=178.4{ }^{\circ} \mathrm{C}($ extr. $), \Delta \mathrm{H}_{\mathrm{v}}=$ $55.00 \mathrm{~kJ} \mathrm{~mol}^{-1}, \Delta \mathrm{S}_{\mathrm{v}}=121.8 \mathrm{~J} \mathrm{~K}^{-1} \mathrm{~mol}^{-1}$.

$\mathrm{C}_{4} \mathrm{Cl}_{2} \mathrm{~F}_{6} \mathrm{~N}_{2} \mathrm{~S}_{2} \quad(\mathrm{M}=325.06)$

$$
\begin{array}{llll}
\text { Calcd } & \text { C 14.8 } & \text { Cl } 21.8 & \text { S 19.7, } \\
\text { Found } & \text { C 14.8 } & \text { Cl 22.0 } & \text { S 19.9. }
\end{array}
$$

MS $m / e$ (cold source) $324 \mathrm{M}^{+}(56)$,

$194 \mathrm{~F}_{3} \mathrm{CC}(\mathrm{Cl})=\mathrm{NS}_{2}+(100), 162 \mathrm{~F}_{3} \mathrm{CC}(\mathrm{Cl})=\mathrm{NS}^{+}(14)$, 99 (65), 93 (15), $69 \mathrm{CF}_{3}{ }^{+}(76), 67(49), 64 \mathrm{~S}_{2}{ }^{+}(68)$. ${ }_{19} \mathrm{~F}$ NMR: $\delta\left(\mathrm{CF}_{3}\right)=-71.0 \mathrm{ppm}(\mathrm{s})\left(40 \%\right.$ in $\left.\mathrm{CFCl}_{3}\right)$. IR (KBr, capillary) $\mathrm{cm}^{-1} .3220$ (w), 1725 (w), $1623(\mathrm{vs}), 1300(\mathrm{~s}, \mathrm{sh}), 1280(\mathrm{vs}), 1255(\mathrm{~s}, \mathrm{sh}), 1205(\mathrm{vs})$, $1160(\mathrm{vs}), 985(\mathrm{~m}, \mathrm{sh}), 945(\mathrm{vs}), 915(\mathrm{~m}, \mathrm{sh}), 773(\mathrm{~s})$, $685(\mathrm{~s}), 543(\mathrm{~m}), 505(\mathrm{~m})$.
10 is pale yellow, $\ln \left(\mathrm{P} / \mathrm{P}_{0}\right)=-6667.0 / \mathrm{T}+13.491$, b. p. $=221.0^{\circ} \mathrm{C}$ (extr.), $\Delta \mathrm{H}_{\mathrm{v}}=55.43 \mathrm{~kJ} \mathrm{~mol}^{-1}$, $\Delta \mathrm{S}_{\mathrm{v}}=112.2 \mathrm{~J} \mathrm{~K}^{-1} \mathrm{~mol}^{-1}$.

$$
\begin{array}{ccc}
\mathrm{C}_{4} \mathrm{Cl}_{2} \mathrm{~F}_{6} \mathrm{~N}_{2} \mathrm{~S}_{3} & (\mathrm{M}=357.15) \\
\text { Calcd } & \mathrm{Cl} 19.9 & \mathrm{~S} 26.9, \\
\text { Found } & \mathrm{Cl} 19.5 & \mathrm{~S} 27.3 .
\end{array}
$$

MS $m / e=356 \mathrm{M}^{+}(49), 292 \mathrm{M}-2 \mathrm{~S}^{+}(22), 257$ (17), $226 \mathrm{~F}_{3} \mathrm{CC}(\mathrm{Cl})=\mathrm{NS}_{3}{ }^{+}(27), 194 \mathrm{~F}_{3} \mathrm{CC}(\mathrm{Cl})=\mathrm{NS}_{2}{ }^{+}(63)$, $162 \mathrm{~F}_{3} \mathrm{CC}(\mathrm{Cl})=\mathrm{NS}^{+}(47), 131(37), 99(48), 69 \mathrm{CF}_{3}{ }^{+}$ (100), 67 (49), $64 \mathrm{~S}_{2}{ }^{+}(66), 57$ (47), 50 (44). ${ }^{19} \mathrm{~F} \mathrm{NMR}$ : $\delta\left(\mathrm{CF}_{3}\right)=-70.8 \mathrm{ppm}(\mathrm{s})\left(30 \%\right.$ in $\left.\mathrm{CFCl}_{3}\right)$. IR $(\mathrm{KBr}$, capillary) $\mathrm{cm}^{-1}: 3215(\mathrm{w}), 1620(\mathrm{~s}), 1300(\mathrm{~s}, \mathrm{sh})$, $1280(\mathrm{vs}), 1253(\mathrm{~s}), 1213(\mathrm{vs}), 1160(\mathrm{vs}), 990(\mathrm{~m})$, $950(\mathrm{vs}), 915(\mathrm{~m}, \mathrm{sh}), 804(\mathrm{w}), 779(\mathrm{~s}), 691(\mathrm{~s}), 549(\mathrm{~m})$, $507(\mathrm{~m})$.

\section{Preparation of $\mathrm{F}_{2} \mathrm{~S}=\mathrm{NC}(\mathrm{Cl})=\mathrm{N}-\mathrm{SCF}_{3}(\mathbf{1 1})$}

$30.60 \mathrm{~g}(0.278 \mathrm{~mol}) 4$ and $40.63 \mathrm{~g}(0.298 \mathrm{~mol}) 2$ were shaken for $17 \mathrm{~d}$ at $40^{\circ} \mathrm{C}$ in a trap with teflon valve, then fractionated repeatedly through a cooled 50 -cm-Vigreux-column. Yield $16.6 \mathrm{~g}(67.3 \mathrm{mmol})=$ $24 \% .11$ is pale yellow, $\ln \left(\mathrm{P} / \mathrm{P}_{0}\right)=-5316.4 / \mathrm{T}+$ 13.157, b. p. $=130.9^{\circ} \mathrm{C}$ (extr.), $\Delta \mathrm{H}_{\mathrm{v}}=44.20 \mathrm{~kJ} \mathrm{~mol}^{-1}$, $\Delta \mathrm{S}_{\mathrm{v}}=109.4 \mathrm{~J} \mathrm{~K}^{-1} \mathrm{~mol}^{-1}$.

\section{$\mathrm{C}_{2} \mathrm{ClF}_{5} \mathrm{~N}_{2} \mathrm{~S}_{2} \quad(\mathrm{M}=246.61)$ \\ Calcd C 9.74 Cl 14.4 N 11.4, \\ Found C 9.88 Cl 14.3 N 11.3.}

$\mathrm{MS} m / e=246 \mathrm{M}^{+}(50), 211 \mathrm{M}-\mathrm{Cl}^{+}(100), 136$ $\mathrm{CF}_{3} \mathrm{SCl}^{+}(31), 101 \mathrm{CF}_{3} \mathrm{~S}^{+}(32), 70(23), 69\left(\mathrm{CF}_{3}{ }^{+}(65)\right.$, $64(33) .{ }^{19} \mathrm{~F} \mathrm{NMR}: \delta\left(\mathrm{CF}_{3}\right)=-50.2(\mathrm{~s}), \delta\left(\mathrm{SF}_{2}\right)=$ $50.6 \mathrm{ppm}$ (s) $\left(50 \%\right.$ in $\left.\mathrm{CFCl}_{3}\right)$. IR (KBr, gas) $\mathrm{cm}^{-1}$ : $1615(\mathrm{~s}), 1393(\mathrm{~m}), 1342(\mathrm{vs}), 1188(\mathrm{vs}), 1138(\mathrm{vs})$, 905 (w, sh), 1018 (m), 848 (m), 812 (w, sh), 750 (s), $708(\mathrm{~s}), 480(\mathrm{w})$.

\section{Preparation of $\mathrm{F}_{2} \mathrm{~S}(\mathrm{O})=\mathrm{NC}(\mathrm{Cl})=\mathrm{N}-\mathrm{SCF}_{3}(\mathbf{1 2})$}

$14.64 \mathrm{~g}(0.116 \mathrm{~mol}) 5$ and $20.27 \mathrm{~g}(0.149 \mathrm{~mol}) 2$ were condensed on $c a .35 \mathrm{ml}$ sulfolane and shaken at $75^{\circ} \mathrm{C}$ for $40 \mathrm{~h}$. The volatile products were separated on a $30-\mathrm{cm}$-Vigreux-column under rough vacuum. Yield $8.10 \mathrm{~g}(30.9 \mathrm{mmol})=27 \%$.

12 is pale yellow, $\ln \left(\mathrm{P} / \mathrm{P}_{0}\right)=-5293.6 / \mathrm{T}+13.561$, b.p. $=117.2^{\circ} \mathrm{C}$ (extr.), $\Delta \mathrm{H}_{\mathrm{v}}=44.01 \mathrm{~kJ} \mathrm{~mol}^{-1}$, $\Delta \mathrm{S}_{\mathrm{v}}=112.7 \mathrm{~J} \mathrm{~K}^{-1} \mathrm{~mol}^{-1}$.

$$
\begin{array}{cccc}
\mathrm{C}_{2} \mathrm{ClF}_{5} \mathrm{~N}_{2} \mathrm{OS}_{2} & (\mathrm{M}=262.61) \\
\text { Calcd } & \mathrm{C} 9.15 & \mathrm{~F} 36.2 & \mathrm{~S} 24.4, \\
\text { Found } & \mathrm{C} 9.22 & \mathrm{~F} 36.0 & \mathrm{~S} 24.7 .
\end{array}
$$

$\mathrm{MS} m / e=262 \mathrm{M}^{+}(72), 227 \mathrm{M}-\mathrm{Cl}^{+}(100), 141$ $\mathrm{M}-\mathrm{SOF}_{2}-\mathrm{Cl}^{+}(85), 103(43), 101 \mathrm{SCF}_{3}{ }^{+}(69), 72(23)$, $69 \mathrm{CF}_{3}{ }^{+}(76), 67(38), 46(61) .{ }^{19} \mathrm{~F} \mathrm{NMR}: \delta\left(\mathrm{CF}_{3}\right)=$ $-50.3(\mathrm{~s}), \delta\left(\mathrm{SOF}_{2}\right)=44.0 \mathrm{ppm}(\mathrm{s})\left(30 \%\right.$ in $\left.\mathrm{CFCl}_{3}\right)$. IR (KBr, gas) $\mathrm{cm}^{-1}$ : 1630 (vs), 1439 (vs), 1349 (w), $1296(\mathrm{vs}), 1230(\mathrm{w}), 1180(\mathrm{vs}), 1137$ (vs), $1049(\mathrm{~m})$, $886(\mathrm{~m}, \mathrm{sh}), 860(\mathrm{vs}), 812(\mathrm{~m}), 797(\mathrm{~m}), 765(\mathrm{w})$, $660(\mathrm{w}), 559(\mathrm{~s}), 446(\mathrm{w}), 415(\mathrm{w})$. 
Preparation of $\mathrm{F}_{2} \mathrm{~S}(\mathrm{O})=\mathrm{NC}(\mathrm{Cl})=\mathrm{N}-\mathrm{SCl}(\mathbf{1 3})$ and $\left(N=C-N=S(O) F_{2}\right)_{3}(\mathbf{1 4})$

$25.34 \mathrm{~g}(0.201 \mathrm{~mol}) 5$ and $23.55 \mathrm{~g}(0.229 \mathrm{~mol}) 1$ were shaken for $11 \mathrm{~d}$ at $45^{\circ} \mathrm{C}$ in a trap with a teflon valve. The volatile products were pumped off on warming, and collected in traps at $0{ }^{\circ} \mathrm{C},-27^{\circ} \mathrm{C}$ and $-196{ }^{\circ} \mathrm{C}$ under oil-pump vacuum. This fractionation was repeated with the $-27{ }^{\circ} \mathrm{C}$ fraction. Yield of $134.10 \mathrm{~g}(17.9 \mathrm{mmol})=9 \%$. The solid reaction residue was sublimed at $35{ }^{\circ} \mathrm{C}$ under oilpump vacuum, then repeatedly recrystallised from $n$-pentane (crystallisation required temperatures down to $\left.-30^{\circ} \mathrm{C}\right)$. Yield of $1412.70 \mathrm{~g}(33.6 \mathrm{mmol})=$ $50 \%$.

13 is orange-yellow, $\ln \left(\mathrm{P} / \mathrm{P}_{0}\right)=-6591.3 / \mathrm{T}+$ 15.880, b. p. $=141.9^{\circ} \mathrm{C}$ (extr.), $\Delta \mathrm{H}_{\mathrm{v}}=54.80 \mathrm{~kJ} \mathrm{~mol}^{-1}$, $\Delta \mathrm{S}_{\mathrm{v}}=132.0 \mathrm{~J} \mathrm{~K}^{-1} \mathrm{~mol}^{-1}$. (13) decomposes slowly in the cold and rapidly at $70^{\circ} \mathrm{C}$.

$$
\begin{array}{cccc}
\mathrm{CCl}_{2} \mathrm{~F}_{2} \mathrm{~N}_{2} \mathrm{OS}_{2} & (\mathrm{M}=229.06) \\
\text { Calcd } & \mathrm{Cl} 31.0 & \mathrm{~N} 12.2 & \mathrm{~S} 28.0, \\
\text { Found } & \mathrm{Cl} 32.2 & \mathrm{~N} 12.0 & \mathrm{~S} 25.8 .
\end{array}
$$

MS (cold source) $m / e=228 \mathrm{M}^{+}(51), 212$ $\mathrm{CClF}_{3} \mathrm{~N}_{2} \mathrm{OS}_{2}{ }^{+}$(25), $193 \mathrm{M}-\mathrm{Cl}^{+}, \mathrm{CClF}_{2} \mathrm{~N}_{2} \mathrm{OS}_{2}{ }^{+}$(40), $177 \mathrm{CF}_{3} \mathrm{~N}_{2} \mathrm{OS}_{2}+(26), 150(31), 126 \mathrm{~F}_{2} \mathrm{~S}(\mathrm{O}) \mathrm{NCN}^{+}(64)$, $107 \mathrm{CClN}_{2} \mathrm{~S}^{+}(100), 102(28), 86 \mathrm{~F}_{2} \mathrm{SO}^{+}(55), 81(21)$, 72 (41), 70 (27), 67 (97), 48 (38), 46 (92). ${ }^{19} \mathrm{~F}$ NMR: $\delta\left(\mathrm{SOF}_{2}\right)=54.3 \mathrm{ppm}(\mathrm{s})\left(60 \%\right.$ in $\left.\mathrm{CFCl}_{3}\right)$. IR ( KBr, gas) $\mathrm{cm}^{-1}$ : $1612(\mathrm{~m}), 1450(\mathrm{~s}, \mathrm{sh}), 1435(\mathrm{~s}), 1295(\mathrm{vs})$, $1140(\mathrm{~m}), 1110(\mathrm{~m}), 1065(\mathrm{w}), 1028(\mathrm{w}), 910(\mathrm{w})$, 880 (w, sh), 847 (s), $808(\mathrm{w}), 560(\mathrm{w}), 510(\mathrm{w})$.

14 is colourless, m.p. $=42{ }^{\circ} \mathrm{C}$, decomp. at $259^{\circ} \mathrm{C}$, dissolves well in $\mathrm{CH}_{2} \mathrm{Cl}_{2}$, slightly in $n$-pentane and $\mathrm{SO}_{2}$, and is insoluble in water.

$$
\begin{array}{cccc}
\mathrm{C}_{3} \mathrm{~F}_{6} \mathrm{~N}_{6} \mathrm{O}_{3} \mathrm{~S}_{3} & (\mathrm{M}=378.25) \\
\text { Calcd } & \mathrm{C} 9.53 & \mathrm{~N} 22.2 & \mathrm{~S} 25.4 \\
\text { Found } & \mathrm{C} 9.60 & \mathrm{~N} 22.3 & \mathrm{~S} \mathrm{25.4}
\end{array}
$$

MS $m / e=378 \mathrm{M}^{+}(81), 252\left[\mathrm{NCNSOF}_{2}\right]_{2}{ }^{+}(38)$, $152(16), 126 \mathrm{NCNSOF}_{2}+(100), 86 \mathrm{SOF}_{2}{ }^{+}(23), 67(24)$, $66(24) .19 \mathrm{~F} \mathrm{NMR}: \delta\left(\mathrm{SOF}_{2}\right)=45.9 \mathrm{ppm}(\mathrm{s})(20 \%$ in $\mathrm{CH}_{2} \mathrm{Cl}_{2} / \mathrm{CFCl}_{3}$ ). IR (KBr, Kel-F/Nujol mulls) $\mathrm{cm}^{-1}$ : $1670(\mathrm{w}), 1620(\mathrm{w}), 1557(\mathrm{~s}), 1543(\mathrm{~s}), 1528(\mathrm{~s}), 1493(\mathrm{~s})$, 1400 (vs, br), 1252 (s), 1238 (s), 1187 (s), 1170 (s), $990(\mathrm{~m}), 978(\mathrm{~m}), 956(\mathrm{~s}), 878(\mathrm{~s}), 847(\mathrm{~s}), 820(\mathrm{~s})$, $792(\mathrm{~s}), 730(\mathrm{~m}, \mathrm{sh}), 665(\mathrm{w}), 635(\mathrm{w}), 592(\mathrm{~m}), 573(\mathrm{~s})$, $560(\mathrm{~s}), 500(\mathrm{~m}), 462(\mathrm{~m}), 439(\mathrm{~m})$.

\section{$X$-ray structural analysis of $\mathbf{( 1 4 )}$}

14 usually crystallises as large fragile plates which are unsuitable for X-ray diffraction. Eventually a suitable crystal was grown by cooling a saturated $n$-pentane solution over a period of 6 weeks. In view of the low melting point data were collected at $173 \mathrm{~K}$ on a Syntex $\mathrm{P} 2_{1}$ 4-circle diffractometer. $\mathrm{Lp}$ and semi-empirical absorption corrections were applied.

9337 unique data were measured, of which 8290 with $\mathrm{F}>4 \sigma(\mathrm{F})$ were used for all calculations.

\section{Crystal data}

$\mathrm{C}_{3} \mathrm{~F}_{6} \mathrm{~N}_{6} \mathrm{O}_{3} \mathrm{~S}_{3}$, triclinic, $\mathrm{P} \overline{1}, a=1133.6(5), b=$ 1332.6(5), $c=1715.1(8) \mathrm{pm}, \alpha=109.31(3), \beta=$ $105.30(3), \gamma=97.84(3)$ at $170{ }^{\circ} \mathrm{K}, \mathrm{U}=2.2862 \mathrm{~nm}^{3}$ $Z=8, \mathrm{D}_{\mathrm{c}}=2.20 \mathrm{Mg} \mathrm{m}^{-3}, \mathrm{~F}(000)=1488$, Mo $\left(\mathrm{K}_{\alpha}\right)$ radiation, $\lambda=71.069 \mathrm{pm}, \mu=0.725 \mathrm{~mm}^{-1}$.

The structure was solved by random-start multisolution tangent refinement followed by Fourier recycling, and refined with all atoms anisotropic and complex, neutral atom scattering factors to $R^{\prime}=\Sigma_{\mathrm{w}^{1 / 2}} \Delta / \Sigma_{\mathrm{w}^{1 / 2}}\left|\mathrm{~F}_{0}\right|=0.037$ and $R=0.028$. The weighting scheme was $\mathrm{w}=\left[\sigma^{2}(\mathrm{~F})+0.0003 \mathrm{~F}^{2}\right]^{-1}$. The results are presented in Tables I and II. Tables

\begin{tabular}{|c|c|c|c|}
\hline Atom & $x$ & $y$ & $z$ \\
\hline$S(1 a)$ & $12817(1)$ & $8577(1)$ & $3734(1)$ \\
\hline $\mathrm{S}(2 \mathrm{a})$ & 14262(1) & $8292(1)$ & $1108(1)$ \\
\hline $\mathrm{S}(3 \mathrm{a})$ & $9703(1)$ & $3676(1)$ & $898(1)$ \\
\hline $\mathrm{S}(1 \mathrm{~b})$ & $107(1)$ & $6086(1)$ & $4019(1)$ \\
\hline $\mathrm{S}(2 \mathrm{~b})$ & $-3004(1)$ & $1150(1)$ & $1260(1)$ \\
\hline $\mathrm{S}(3 \mathrm{~b})$ & $-4518(1)$ & $1565(1)$ & $3867(1)$ \\
\hline $\mathrm{S}(1 \mathrm{c})$ & $3686(1)$ & $4709(1)$ & $3436(1)$ \\
\hline $\mathrm{S}(2 \mathrm{c})$ & $725(1)$ & $365(1)$ & $3650(1)$ \\
\hline $\mathrm{S}(3 \mathrm{c})$ & $3322(1)$ & $2454(1)$ & $6239(1)$ \\
\hline$S(1 d)$ & $4243(1)$ & $5300(1)$ & $-1465(1)$ \\
\hline$S(2 d)$ & $8263(1)$ & $7384(1)$ & $1233(1)$ \\
\hline$S(3 d)$ & $8946(1)$ & $9534(1)$ & $-1549(1)$ \\
\hline$C(1 a)$ & $12093(2)$ & $6873(2)$ & $2340(1)$ \\
\hline $\mathrm{C}(2 \mathrm{a})$ & $12785(2)$ & $6741(2)$ & $1218(1)$ \\
\hline $\mathrm{C}(3 \mathrm{a})$ & $11377(2)$ & $5344(2)$ & $1139(1)$ \\
\hline$C(1 b)$ & $-1570(2)$ & $4443(2)$ & $3815(1)$ \\
\hline $\mathrm{C}(2 \mathrm{~b})$ & $-2272(2)$ & $2886(2)$ & $2642(1)$ \\
\hline$C(3 b)$ & $-2950(2)$ & $3060(2)$ & $3779(1)$ \\
\hline $\mathrm{C}(1 \mathrm{c})$ & $2217(2)$ & $3302(1)$ & $3691(1)$ \\
\hline $\mathrm{C}(2 \mathrm{c})$ & $821(2)$ & $2058(2)$ & $3802(1)$ \\
\hline $\mathrm{C}(3 \mathrm{c})$ & $2656(2)$ & $2898(2)$ & $4880(1)$ \\
\hline$C(1 d)$ & $5889(2)$ & $6933(2)$ & $-1298(1)$ \\
\hline $\mathrm{C}(2 \mathrm{~d})$ & $7678(2)$ & $7762(2)$ & $-172(1)$ \\
\hline$C(3 d)$ & $7362(2)$ & $8151(2)$ & $-1367(1)$ \\
\hline $\mathrm{N}(1 \mathrm{a})$ & $12827(1)$ & $7369(1)$ & $2015(1)$ \\
\hline $\mathrm{N}(2 \mathrm{a})$ & $12086(2)$ & $5724(1)$ & $738(1)$ \\
\hline$N(3 a)$ & $11338(1)$ & $5862(1)$ & $1938(1)$ \\
\hline $\mathrm{N}(4 \mathrm{a})$ & $12091(2)$ & $7427(1)$ & $3185(1)$ \\
\hline$N(5 a)$ & $13544(2)$ & $7147(1)$ & $813(1)$ \\
\hline$N(6 a)$ & $10623(2)$ & $4283(1)$ & $631(1)$ \\
\hline$N(1 b)$ & $-1535(2)$ & $3898(1)$ & $3021(1)$ \\
\hline$N(2 b)$ & - 3002(1) & $2409(1)$ & $2985(1)$ \\
\hline $\mathrm{N}(3 \mathrm{~b})$ & $-2255(2)$ & $4086(1)$ & $4240(1)$ \\
\hline$N(4 b)$ & $819(2)$ & $5514(1)$ & $4302(1)$ \\
\hline$N(5 b)$ & $-2262(2)$ & $2295(1)$ & $1801(1)$ \\
\hline $\mathrm{N}(6 \mathrm{~b})$ & $-3688(2)$ & $2675(1)$ & $4206(1)$ \\
\hline$N(1 c)$ & $1074(1)$ & $2613(1)$ & $3324(1)$ \\
\hline $\mathrm{N}(2 \mathrm{c})$ & $1566(1)$ & $2146(1)$ & $4579(1)$ \\
\hline$N(3 c)$ & $3054(1)$ & $3494(1)$ & $4470(1)$ \\
\hline $\mathrm{N}(4 \mathrm{c})$ & $2523(1)$ & $3842(1)$ & $3175(1)$ \\
\hline $\mathrm{N}(5 \mathrm{c})$ & $366(1)$ & $1313(1)$ & $3419(1)$ \\
\hline$N(6 c)$ & $3506(1)$ & $3106(1)$ & $5709(1)$ \\
\hline$N(1 d)$ & $6562(1)$ & $7033(1)$ & $-499(1)$ \\
\hline
\end{tabular}
of thermal parameters, structure factors, bond lengths and angles may be obtained from the authors.

Table I. Atomic co-ordinates $\left(\times 10^{4}\right)$ with estimated standard deviations in parentheses. 
Table I (continued).

\begin{tabular}{|c|c|c|c|}
\hline Atom & $x$ & $y$ & $z$ \\
\hline$N(2 d)$ & $8137(1)$ & $8341(1)$ & $-572(1)$ \\
\hline$N(3 d)$ & $6219(1)$ & $7465(1)$ & $-1771(1)$ \\
\hline$N(4 d)$ & $4694(1)$ & $6201(1)$ & $-1720(1)$ \\
\hline$N(5 d)$ & $8479(2)$ & $7972(1)$ & $666(1)$ \\
\hline$N(6 d)$ & $7737(1)$ & $8697(1)$ & $-1862(1)$ \\
\hline$O(1 \mathrm{a})$ & $12716(1)$ & $9053(1)$ & $4562(1)$ \\
\hline$O(2 a)$ & $14923(1)$ & $8545(1)$ & $586(1)$ \\
\hline $\mathrm{O}(3 \mathrm{a})$ & $8972(1)$ & $2643(1)$ & $283(1)$ \\
\hline $\mathrm{O}(1 \mathrm{~b})$ & $820(1)$ & $7137(1)$ & $4605(1)$ \\
\hline $\mathrm{O}(2 \mathrm{~b})$ & - 2885(1) & $640(1)$ & $440(1)$ \\
\hline $\mathrm{O}(3 \mathrm{~b})$ & $-5228(1)$ & 1334(1) & $4372(1)$ \\
\hline $\mathrm{O}(\mathrm{lc})$ & $3813(1)$ & $5156(1)$ & $2820(1)$ \\
\hline $\mathrm{O}(2 \mathrm{c})$ & $-1879(1)$ & $-395(1)$ & $3125(1)$ \\
\hline $\mathrm{O}(3 \mathrm{c})$ & $4254(1)$ & $2728(1)$ & $7047(1)$ \\
\hline $\mathrm{O}(\mathrm{ld})$ & $3091(1)$ & $4548(1)$ & $-2015(1)$ \\
\hline$O(2 d)$ & $9208(2)$ & $7650(1)$ & 2031(1) \\
\hline$O(3 d)$ & $9175(1)$ & $10015(1)$ & $-2123(1)$ \\
\hline $\mathrm{F}(1 \mathrm{a})$ & $12567(1)$ & $9346(1)$ & $3251(1)$ \\
\hline $\mathrm{F}(2 \mathrm{a})$ & $14217(1)$ & $8749(1)$ & $3826(1)$ \\
\hline $\mathrm{F}(3 \mathrm{a})$ & $15175(1)$ & $8740(1)$ & $2049(1)$ \\
\hline$F(4 a)$ & $13449(1)$ & $9109(1)$ & $1305(1)$ \\
\hline$F(5 a)$ & $8838(1)$ & $4361(1)$ & $1236(1)$ \\
\hline $\mathrm{F}(6 \mathrm{a})$ & $10324(1)$ & $3565(1)$ & $1757(1)$ \\
\hline$F(1 b)$ & $986(1)$ & $5388(1)$ & $3724(1)$ \\
\hline$F(2 b)$ & 495(1) & $6133(1)$ & $3129(1)$ \\
\hline$F(3 b)$ & $-4411(1)$ & $1019(1)$ & $1144(1)$ \\
\hline$F(4 b)$ & $-2810(1)$ & $384(1)$ & $1753(1)$ \\
\hline$F(5 b)$ & $-3813(1)$ & $668(1)$ & $3640(1)$ \\
\hline$F(6 b)$ & $-5396(1)$ & $1200(1)$ & 2926(1) \\
\hline$F(1 c)$ & 4891(1) & $4369(1)$ & $3764(1)$ \\
\hline$F(2 c)$ & $3885(1)$ & $5642(1)$ & $4299(1)$ \\
\hline$F(3 c)$ & $315(1)$ & $-252(1)$ & $3738(1)$ \\
\hline$F(4 c)$ & $-\quad 666(1)$ & $693(1)$ & $4605(1)$ \\
\hline$F(5 c)$ & $2040(1)$ & 2391(1) & $6380(1)$ \\
\hline$F(6 c)$ & $3068(1)$ & $1223(1)$ & $5716(1)$ \\
\hline$F(1 d)$ & $4202(1)$ & $5702(1)$ & $-533(1)$ \\
\hline$F(2 d)$ & $5247(1)$ & $4674(1)$ & $-1287(1)$ \\
\hline$F(3 d)$ & $7891(1)$ & $6144(1)$ & $746(1)$ \\
\hline$F(4 d)$ & 7016(1) & $7449(1)$ & $1411(1)$ \\
\hline$F(5 d)$ & $9127(1)$ & $10439(1)$ & $-676(1)$ \\
\hline$F(6 d)$ & $10091(1)$ & $9126(1)$ & $-1210(1)$ \\
\hline
\end{tabular}

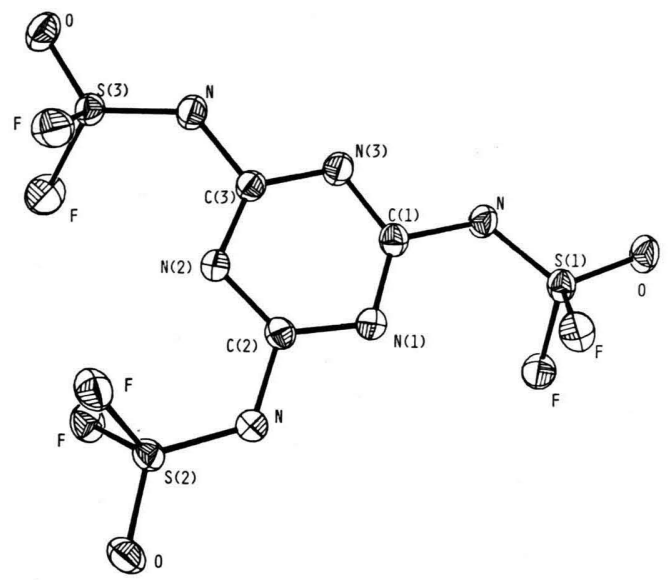

Figure. Molecule B of 14, showing 50\% probability thermal allipsoids and the labelling scheme used for the mean bond lengths and angles (Table II). The other three independent molecules are very similar.

\section{Results and Discussion}

\section{A. Additions to $\mathrm{CF}_{3} \mathrm{CN}(\mathbf{3})$}

The reaction of 2 with 3 has already been reported [4]:

$$
\begin{aligned}
& \underset{2}{\mathrm{CF}_{3} \mathrm{SCl}}+\underset{3}{\mathrm{CF}_{3} \mathrm{CN}} \underset{12 \mathrm{~h}}{\stackrel{\mathrm{h} v}{\longrightarrow}} \\
& \underset{6}{\mathrm{CF}_{3} \mathrm{~S}-\mathrm{N}=\mathrm{C}^{\mathrm{C}}} \stackrel{\mathrm{CF}_{3}}{\mathrm{Cl}} \quad(30 \%) \\
& 2+3 \underset{18 \mathrm{~h} / 50{ }^{\circ} \mathrm{C}}{\longrightarrow} \rightarrow \quad(50 \%)
\end{aligned}
$$

Table II. Mean bond lengths (pm) and angles $\left({ }^{\circ}\right)$ for (14) using the notation in the Figure. Standard deviations have been calculated from the deviations of the individual values from the mean. Estimated standard deviations

\begin{tabular}{|c|c|c|c|c|c|}
\hline $\begin{array}{l}\mathrm{S}(1)-\mathrm{O} \\
\mathrm{S}(1)-\mathrm{F} \\
\mathrm{S}(1)-\mathrm{N} \\
\mathrm{N}-\mathrm{C}(1) \\
\mathrm{C}(1)-\mathrm{N}(1) \\
\mathrm{C}(1)-\mathrm{N}(3)\end{array}$ & $\begin{array}{l}140.1(2) \\
152.3(2) \\
148.7(3) \\
139.6(4) \\
133.3(4) \\
133.5(1)\end{array}$ & $\begin{array}{l}\mathrm{S}(2)-\mathrm{O} \\
\mathrm{S}(2)-\mathrm{F} \\
\mathrm{S}(2)-\mathrm{N} \\
\mathrm{N}-\mathrm{C}(2) \\
\mathrm{C}(2)-\mathrm{N}(2) \\
\mathrm{C}(2)-\mathrm{N}(1)\end{array}$ & $\begin{array}{l}139.7(2) \\
152.9(4) \\
148.3(1) \\
139.8(5) \\
133.7(3) \\
133.1(4)\end{array}$ & $\begin{array}{l}\mathrm{S}(3)-\mathrm{O}) \\
\mathrm{S}(3)-\mathrm{F} \\
\mathrm{S}(3)-\mathrm{N} \\
\mathrm{N}-\mathrm{C}(3) \\
\mathrm{C}(3)-\mathrm{N}(3) \\
\mathrm{C}(3)-\mathrm{N}(2)\end{array}$ & $\begin{array}{l}140.2(2) \\
152.9(3) \\
147.8(3) \\
140.0(3) \\
133.1(3) \\
133.3(2)\end{array}$ \\
\hline $\begin{array}{l}\mathrm{O}-\mathrm{S}(1)-\mathrm{F} \\
\mathrm{O}-\mathrm{S}(1)-\mathrm{N} \\
\mathrm{F}-\mathrm{S}(1)-\mathrm{N} \\
\mathrm{S}(1)-\mathrm{N}-\mathrm{C} \\
\mathrm{F}-\mathrm{S}(1)-\mathrm{F} \\
\mathrm{N}-\mathrm{C}(1)-\mathrm{N}(1) \\
\mathrm{N}-\mathrm{C}(1)-\mathrm{N}(3) \\
\mathrm{N}(1)-\mathrm{C}-\mathrm{N}(3) \\
\mathrm{C}-\mathrm{N}(1)-\mathrm{C}\end{array}$ & $\begin{array}{r}108.8(3) \\
117.0(2) \\
112.4(5) \\
124.8(6) \\
95.4(2) \\
119.6(2) \\
113.4(4) \\
127.0(4) \\
113.4(3)\end{array}$ & $\begin{array}{l}\mathrm{O}-\mathrm{S}(2)-\mathrm{F} \\
\mathrm{O}-\mathrm{S}(2)-\mathrm{N} \\
\mathrm{F}-\mathrm{S}(2)-\mathrm{N} \\
\mathrm{S}(2)-\mathrm{N}-\mathrm{C} \\
\mathrm{F}-\mathrm{S}(2)-\mathrm{F} \\
\mathrm{N}-\mathrm{C}(2)-\mathrm{N}(2) \\
\mathrm{N}-\mathrm{C}(2)-\mathrm{N}(1) \\
\mathrm{N}(2)-\mathrm{C}-\mathrm{N}(1) \\
\mathrm{C}-\mathrm{N}(2)-\mathrm{C}\end{array}$ & $\begin{array}{c}108.4(3) \\
118.1(2) \\
112.3(3) \\
124.0(11) \\
95.0(2) \\
119.3(5) \\
114.2(3) \\
126.4(3) \\
113.4(2)\end{array}$ & $\begin{array}{l}\mathrm{O}-\mathrm{S}(3)-\mathrm{F} \\
\mathrm{O}-\mathrm{S}(3)-\mathrm{N} \\
\mathrm{F}-\mathrm{S}(3)-\mathrm{N} \\
\mathrm{S}(3)-\mathrm{N}-\mathrm{C} \\
\mathrm{F}-\mathrm{S}(3)-\mathrm{F} \\
\mathrm{N}-\mathrm{C}(3)-\mathrm{N}(2) \\
\mathrm{N}-\mathrm{C}(3)-\mathrm{N}(3) \\
\mathrm{N}(3)-\mathrm{C}-\mathrm{N}(2) \\
\mathrm{C}-\mathrm{N}(3)-\mathrm{C}\end{array}$ & $\begin{array}{l}108.4(5) \\
117.9(1) \\
112.7(7) \\
125.3(10) \\
94.4(4) \\
119.4(2) \\
113.7(2) \\
127.0(1) \\
112.9(2)\end{array}$ \\
\hline
\end{tabular}
from the least-squares refinement were $0.1^{\circ}$ for individual angles, $0.2 \mathrm{pm}$ for bonds involving $\mathrm{S}$ and $0.3 \mathrm{pm}$ for other bonds. 
We were not able to reproduce these quoted yields, especially for the thermal reaction. However, when the reaction was carried out in the absence of solvent but with active charcoal as catalyst, the desired product 6 could be isolated in $82 \%$ yield. The reaction of $\mathbf{1}$ is analogous:

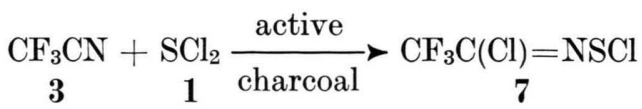

1 tends to poison the catalyst, which if necessary must be renewed during the reaction. It is advisable to use only a slight excess of $\mathbf{1}$, since otherwise appreciable quantities of $\mathrm{CF}_{3} \mathrm{CCl}_{2} \mathrm{~N}=\mathrm{SCl}_{2}$ (8) are formed by addition of $\mathrm{Cl}_{2}$ [in equilibrium with 1 and $\mathrm{S}_{2} \mathrm{Cl}_{2}$ ] to 7.8 is stable in glass vessels, but can be dehalogenated to 7 by metals (e.g. the steel parts of GC-columns). Mercury reacts slowly with the $\mathrm{S}-\mathrm{Cl}$ bond of $\mathbf{7}$, forming $\mathrm{Hg}_{2} \mathrm{Cl}_{2}$, the disulphane $\left(\mathrm{CF}_{3} \mathrm{C}(\mathrm{Cl})=\mathrm{N}-\mathrm{S}\right)_{2}(\mathbf{9})$ in $30 \%$ and the trisulphane $\left(\mathrm{CF}_{3} \mathrm{C}(\mathrm{Cl})=\mathrm{N}-\mathrm{S}\right)_{2} \mathrm{~S}(\mathbf{1 0})$ in $13 \%$ yield. Both are stable to excess mercury.

\section{B. Additions to $\mathrm{NCNSF}_{2}(4)$}

2 may be added thermally to the triple bond of 4 :

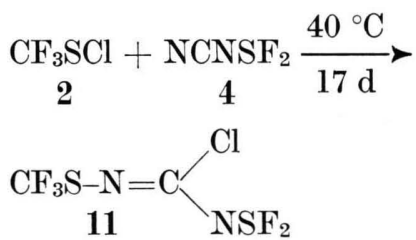

11 is isolated in $24 \%$ yield, the reaction mixture consisting primarily of starting materials which are probably in equilibrium with 11. Higher tempera-

Table III. Infrared frequencies $\left(\mathrm{cm}^{-1}\right)$ for N-sulphenylimidoyl derivatives.

\begin{tabular}{llll}
\hline Compound & $v(\mathrm{C}=\mathrm{N})$ & $\begin{array}{l}\text { Inten- } \\
\text { sity }\end{array}$ & Literature \\
\hline $\mathrm{ClS}-\mathrm{N}=\mathrm{CCl}_{2}$ & 1594 & $\mathrm{~s}$ & 6 \\
$\mathrm{CF}_{3} \mathrm{~S}-\mathrm{N}=\mathrm{CCl}_{2}$ & 1614 & vs & 7 \\
$\mathrm{~F}_{5} \mathrm{~S}-\mathrm{N}=\mathrm{CCl}_{2}$ & 1653 & & 15 \\
$\mathrm{ClS}-\mathrm{N}=\mathrm{C}(\mathrm{Cl}) \mathrm{CF}_{3}$ & 1624 & $\mathrm{~s}$ & this work \\
$\left(-\mathrm{S}-\mathrm{N}=\mathrm{C}(\mathrm{Cl}) \mathrm{CF}_{3}\right)_{2}$ & 1623 & vs & this work \\
$\mathrm{S}\left(-\mathrm{S}-\mathrm{N}=\mathrm{C}(\mathrm{Cl}) \mathrm{CF}_{3}\right)_{2}$ & 1620 & $\mathrm{~s}$ & this work \\
$\mathrm{ClS}-\mathrm{N}=\mathrm{C}\left(\mathrm{CF}_{3}\right)_{2}$ & 1630 & $\mathrm{~m}$ & 2 \\
$\left(-\mathrm{S}-\mathrm{N}=\mathrm{C}\left(\mathrm{CF}_{3}\right)_{2}\right)_{2}$ & 1630 & $\mathrm{w}$ & 2 \\
$\mathrm{CF}$ & 1655 & $\mathrm{w}$ & 16 \\
$\left.\mathrm{FS}-\mathrm{N}=\mathrm{C}(\mathrm{CF})_{3}\right)_{2}$ & 1620 & $\mathrm{~W}$ & 17 \\
$\mathrm{ClS}-\mathrm{N}=\mathrm{C}(\mathrm{Cl}) \mathrm{N}=\mathrm{S}(\mathrm{O}) \mathrm{F}_{2}$ & 1612 & $\mathrm{~m}$ & this work \\
$\mathrm{CF}$ & $16 \mathrm{~S}-\mathrm{N}=\mathrm{C}(\mathrm{Cl}) \mathrm{N}=\mathrm{S}(\mathrm{O}) \mathrm{F}_{2} 1630$ & $\mathrm{vs}$ & this work \\
$\mathrm{CF}_{3} \mathrm{~S}-\mathrm{N}=\mathrm{C}(\mathrm{Cl}) \mathrm{N}=\mathrm{SF}_{2}$ & 1615 & $\mathrm{~s}$ & this work \\
\hline
\end{tabular}

ture led to decomposition of 4 . It is possible to obtain pure 11 by low temperature vacuum distillation; it is stable for a short time up to $100{ }^{\circ} \mathrm{C}$.

$\mathrm{N}$-Sulphenylimidoyl derivatives such as $\mathbf{1 1}$ may be unambiguously identified by NMR and massspectra, and in particular by $v(\mathrm{CN})$ in the IR, which may be found with varying intensity in the range 1600 to $1650 \mathrm{~cm}^{-1}$ (Table III).

Electronegative substituents on $\mathrm{C}$ raise $v(\mathrm{CN})$, which lies about $20-30 \mathrm{~cm}^{-1}$ higher in the $\mathrm{CF}_{3} \mathrm{~S}$ derivatives than in the corresponding sulphenyl chlorides.

The reaction of $\mathrm{SCl}_{2}(\mathbf{1})$ with 4 follows a completely different path, with the formation of 4-chloro-1,2dithia-3,5-diazolium chloride [18].

\section{Additions to $\mathrm{NC}-\mathrm{NSOF}_{2}(5)$}

$\mathrm{NC}-\mathrm{NSOF}_{2}(\mathbf{5})$ reacts similarly to 4 , but the addition reactions may be carried out at higher temperature because it is thermally more stable.

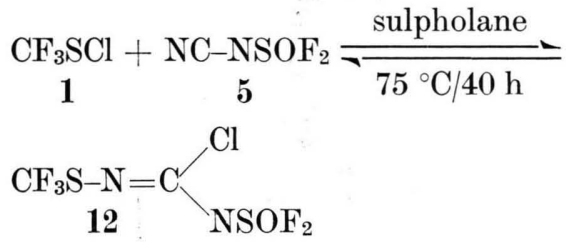

After $40 \mathrm{~h}$ only $27 \%$ conversion is obtained, and longer reaction time does not increase the yield. With $\mathrm{SCl}_{2}(\mathbf{1})$ the desired $\mathrm{N}$-chlorosulphenyl-imidoyl chloride (13) is obtained in low yield. 13 is thermally labile and decomposes slowly even at $-30{ }^{\circ} \mathrm{C}$.

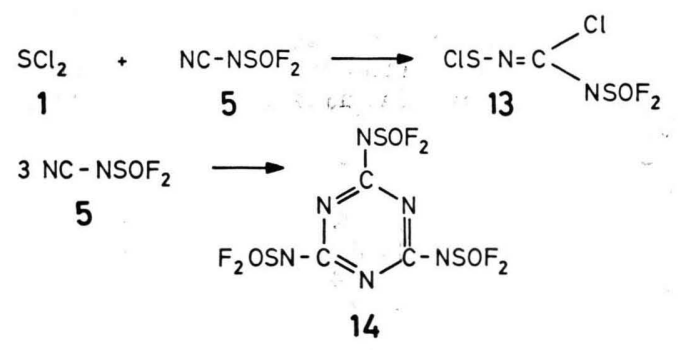

The main product of this reaction is $2,4,6$-tris(difluorosulphoximido)-1,3,5-triazene (14), the cyclic trimer of $\mathbf{5}$. This catalytic action of $\mathbf{1}$ is also observed, to a much smaller extent, in its reactions with $\mathrm{ClCN}[6]$ and $\mathrm{CF}_{3} \mathrm{CN}$ (3), where the corresponding trimers are formed as byproducts. We report 
here the crystal structure of $\mathbf{1 4}$; in view of the low melting point the data were collected at $173^{\circ} \mathrm{K}$.

\section{Crystal structure of ( $\left.\mathrm{NCNSOF}_{2}\right)_{3}(\mathbf{1 4})$}

The four crystallographically independent molecules all adopt the same unsymmetrical conformation, although a $180^{\circ}$ rotation about one $\mathrm{S}-\mathrm{N}$ bond would generate the 'least hindered' symmetrical $\mathrm{C}_{3 \mathrm{~h}}$ conformation. Each molecule possesses an approximate plane of symmetry through all the ring atoms. Two S-O units deviate by about $50 \mathrm{pm}$ from this plane, the others by much less; these deviations are not systematic and reflect small rotations about the exocyclic $\mathrm{C}-\mathrm{N}$ bonds, presumably dictated by packing forces. All $\mathrm{S}-\mathrm{O}$ bonds are trans to $\mathrm{C}-\mathrm{N}$ bonds, and all $\mathrm{N}-\mathrm{C}-\mathrm{N}$ angles $c i$ s to $\mathrm{S}$ are about $5.7^{\circ}$ wider than the trans angles. In Table II the bond lengths and angles have been averaged over the four molecules, assuming an idealised $\mathrm{m}\left(\mathrm{C}_{\mathrm{s}}\right)$ molecular symmetry. The esds (calculated from the deviations of the individual values from the mean) are little greater than the individual least-squares esds $(0.2 \mathrm{pm}$ for bonds involving $\mathrm{S}, 0.3 \mathrm{pm}$ for others; $0.1^{\circ}$ for angles) indicating that the 4 molecules do not differ significantly (except for torsional angles). The differences between the conformationally different $-\mathrm{N}=\mathrm{S}(\mathrm{O}) \mathrm{F}_{2}$ groups are only marginally significant.
The short $\mathrm{S}-\mathrm{O}$ and $\mathrm{S}-\mathrm{N}$ bonds indicate appreciable double-bond character. The mean $\mathrm{N}-\mathrm{C}$ bond length in the ring [133.3(2) pm] is slightly smaller than the mean value of $133.8 \mathrm{pm}$ found in s-triazine [19], 2,4,6-triamino-s-triazine [20] and 2,4,6-tris(dimethylamino)-s-triazine [21] and the mean exocyclic $\mathrm{C}-\mathrm{N}$ distance of $139.8(3) \mathrm{pm}$ is appreciably longer than the values of 134.2 and $135.9 \mathrm{pm}$ resp. found in the latter two compounds [for consistency, all values are quoted without librational correction]. The electron-withdrawing $-\mathrm{SOF}_{2}$ group appears to reduce the back donation of nitrogen lone-pair electrons into the exocyclic $\mathrm{N}-\mathrm{C}$ bonds. In vapour phase $\mathrm{HN}=\mathrm{S}(\mathrm{O}) \mathrm{F}_{2}[22]$ and $\mathrm{ClN}=\mathrm{S}(\mathrm{O}) \mathrm{F}_{2}$ [23] the oxygen atom is also trans to the substituent on nitrogen. Both show longer S-F bonds (154.9 and $154.8 \mathrm{pm}$ resp.) and smaller F-S-F angles (93.7 and $92.6^{\circ}$ resp.) than the values reported here, though this could be accounted for by the affect of torsion about the $\mathrm{S}-\mathrm{N}$ bonds on the apparent $\mathrm{F}$ positions in the crystal. Otherwise the $-\mathrm{NS}(\mathrm{O}) \mathrm{F}_{2}$ group geometry is very similar to that in $\mathrm{ClN}=\mathrm{S}(\mathrm{O}) \mathrm{F}_{2}$; in $\mathrm{HN}=\mathrm{S}(\mathrm{O}) \mathrm{F}_{2}$ the $\mathrm{N}=\mathrm{S}$ bond is about $2 \mathrm{pm}$ shorter, and the $\mathrm{S}=\mathrm{O}$ bond about $2 \mathrm{pm}$ longer. There are no particularly short intermolecular interactions in $\mathbf{1 4}$.

We thank the Verband der Chemischen Industrie for support.
[1] A. Haas, Gmelins Handbuch der anorganischen Chemie, Ergänzungswerk zur achten Auflage, Band 9, Teil 1, p. 157 (1973).

[2] S. G. Metcalf and J. M. Shreeve, Inorg. Chem. 11, 1631 (1972).

[3] H. Steinbeisser, R. Mews, and O. Glemser, Z. Naturforsch. 32 b, 160 (1977).

[4] C. A. Burton and J. M. Shreeve, Inorg. Chem. 16, 1408 (1977).

[5] G. Varwig and R. Mews, J. Chem. Res. 1977, 2744.

[6] R. G. R. Bacon, R. S. Irwin, J. McC. Pollock, and A. D. E. Pullin, J. Chem. Soc. 1958, 764.

[7] P. Gielow and A. Haas, Z. Anorg. Allg. Chem. 394, 53 (1972).

[8] P. Gielow and A. Haas, Chemiker-Ztg. 95, 1010 (1971).

[9] E. Kühle, B. Anders, and G. Zumach, Angew. Chem. 79, 663 (1967); Angew. Chem., Int. Ed. Engl. 6, 649 (1967).

[10] G. Brauer (ed.): Handbuch der präp. anorg. Chemie 1, 380 (1975).

[11] C. W. Tullock and D. D. Coffman, J. Org. Chem. 25, 2016 (1960).

[12] H. Gilman and R. G. Jones, J. Am. Chem. Soc. 65, 1458 (1943).
[13] O. Glemser and M. Biermann, Inorg. Nucl. Chem. Lett. 3, 223 (1967); W. Sundermeyer, Angew. Chem. 79, 98 (1967).

[14] M. Lustig and J. K. Ruff, Inorg. Nucl. Chem. Lett. 3, 531 (1967); O. Glemser, U. Biermann, and A. Hoff, Z. Naturforsch. 22 b, 893 (1967).

[15] C. W. Tullock, D. D. Coffman, and E. L. Muetterties, J. Am. Chem. Soc. 86, 357 (1969).

[16] R. F. Swindell, D. P. Babb, T. J. Quelette, and J. M. Shreeve, Inorg. Chem. 11, 242 (1972).

[17] J. Varwig, H. Steinbeisser, R. Mews, and $O$. Glemser, Z. Naturforsch. 29 b, 813 (1974).

[18] H.-U. Höfs, R. Mews, W. Clegg, M. Noltemeyer, M. Schmidt, and G. M. Sheldrick, Chem. Ber. 116, 416 (1983).

[19] J. H. Smith and A. I. M. Rae, J. Phys. Chem. 11, 1761 (1978).

[20] A. C. Larson and D. T. Cromer, J. Chem. Phys. 60, 185 (1974).

[21] G. J. Bullen, D. J. Corney, and F. S. Stephens, J. Chem. Soc. Perkin Trans. 2 1972, 642.

[22] P. Cassoux, R. L. Kuczkowski, and R. A. Creswell, Inorg. Chem. 16, 2959 (1977).

[23] O. Oberhammer, O. Glemser, and H. Klüver, Z. Naturforsch. 29 a, 901 (1974). 